\title{
ROBOTIC ADENOMASTECTOMY: FOLLOW-UP OF OPERATED CASES
}

Rodrigo Ferreira Bernardi', José Clemente Linhares', Audrey Tieko Tsunoda', Anne Groth¹, Sergio Bruno Bonatto Hatschbach ${ }^{1}$

${ }^{1}$ Hospital Erasto Gaertner - Curitiba (PR), Brazil.

Introduction: For the past few years, Nipple-Sparing Mastectomy (NSM) has been a tendency, and robotic breast surgery has become a reality. Until August, 2019, three robotic adenomastectmy procedures were carried out in Hospital Erasto Gaertner. The objective of this article is to demonstrate our results and publicize the knowledge in robotic surgery. Methods: Surgical Technique. The Da Vinci Surgical System Si (Intuitive Surgical, Sunnyvale, CA) was used to perform the NSM surgeries. The $3 \mathrm{~cm}$ long incision was initiated in the axillary region, and the subcutaneous cellular tissue was resected to an area of $5 \mathrm{~cm}$ in diameter, to the portal. One $\mathrm{mg} / \mathrm{ml}$ of adrenaline diluted in physiological serum $0.9 \%$ was infiltered in the subdermal breast tissue. Tunneling is executed with Metzenbaum scissors in the subdermal region. The device is coupled (GelPOINT Advanced Access Platform ${ }^{\mathrm{tm}}$ ), and gas pressure of $8 \mathrm{mmHg}$ is activated. The ProGrasp Forceps, the monopolar curved scissors and the $30^{\circ}$ video camera are allocated in the robotic arms. The upper quadrants are superficially dissected, and then the lower quadrants, the retro-mammillary region and, finally, the breast muscle tissue. The expander is allocated between the chests with the open technique. Result: The duration of the mammary gland extirpation was 5 hours in the first unilateral adenomastectomy surgery, and 1 hour and 45 minutes in the last for the same type of surgery. In total, the first surgery took 6 hours and 20 minutes, which reduced to 3 hours in the last. In two surgeries we observed skin burns caused by electrocautery. These were solved in weeks, however, without the need for surgical intervention. We did not observe complications such as seroma, hematoma, loss of prosthesis, major pain, infection or subcutaneous emphysema due to carbon dioxide. Discussion: Complication rates are low, as shown by Lai et al. (2019), who analyzed a case series and showed transient nipple ischemia (10.3\%) as the highest rate of complication; however, without nipple necrosis or loss of prosthesis. In the cases operated in Hospital Erasto Gaertner, the aesthetic results were very well evaluated both by the patients and the plastic surgery and mastology staff. Minor complications were solved in weeks, which maintained an excellent final outcome. The learning curve of the robotic technique was extremely fast, with considerable reduction in surgical time. Conclusion: We believe that innovative robotic surgery can contribute with the advancement of breast surgery around the world. 